\title{
HYGROTHERMAL DURABILITY OF BOND IN FRP-STRENGTHENED MASONRY
}

\author{
Bahman Ghiassi' ${ }^{1}$, Daniel V. Oliveira ${ }^{2}$, Paulo B. Lourenço ${ }^{3}$ \\ ISISE, University of Minho, Department of Civil Engineering, Guimarães, \\ Portugal
}

\begin{abstract}
Fiber reinforced polymers (FRPs) are accepted as an efficient material for external strengthening of masonry structures. Previous researches have shown that the bond between FRP and the substrate plays an important role in the effectiveness of this strengthening technique. Extensive investigations have been devoted to the characterization of the short-term bond behavior, while its durability and long-term performance requires further studies. In this regard, a full experimental program for investigating the environmental durability of bond in FRP-strengthened masonry is crucial for understanding the degrading mechanisms.

This paper presents the results of an experimental program aimed at investigating the hygrothermal durability of bond in FRP-strengthened bricks. Accelerated ageing tests were performed on the FRP-strengthened brick elements and the bond degradation was periodically investigated by visual inspection and by conventional single-lap shear bond tests. The changes in the properties of material constituents have also been monitored. The obtained results are presented and critically discussed.
\end{abstract}

Keywords: FRP; masonry; bond; durability; hygrothermal; accelerated ageing.

\footnotetext{
${ }^{1}$ Postdoctoral researcher, ISISE, University of Minho, Department of Civil Engineering, Azurém, 4800-058 Guimarães, Portugal.

Phone: +351253510499

Fax: +351253510217

E-mail: bahmanghiassi@civil.uminho.pt

${ }^{2}$ Associate Professor, ISISE, University of Minho, Department of Civil Engineering, Azurém, 4800-058 Guimarães, Portugal.

Phone: +351253510247

Fax: +351 253510217

E-mail: danvco@civil.uminho.pt

${ }^{3}$ Professor, ISISE, University of Minho, Department of Civil Engineering, Azurém, 4800-058 Guimarães, Portugal.

Phone: +351 253510209

Fax: +351253510217

E-mail: pbl@ civil.uminho.pt
} 


\section{Introduction}

Fiber reinforced polymers (FRPs) are accepted as an efficient material for external strengthening of masonry structures. Advantages such as their light weight, high mechanical properties and easy processing have made this material a suitable choice for strengthening purposes. This strengthening technique is usually applied following a wet lay-up procedure in which the fibers are saturated with epoxy resin and applied directly on the substrate's surface. The efficacy and reliability of this strengthening technique depends intrinsically on the bond between the composite material and masonry substrate. The short-term bond behavior have been extensively investigated in the last years, e.g. (Grande et al. 2008; Faella et al. 2012; Capozucca 2012; Ghiassi et al. 2012a; Ghiassi et al. 2012b; Valluzzi et al. 2012). Another important aspect of the bond behavior is its long-term performance. Investigating the long-term durability of the bond behavior is of crucial importance in service life prediction and structural safety evaluation (Karbhari et al. 2003; Hollaway 2010). However, only a few studies can be found in the literature devoted to the durability of bond in FRP-strengthened masonry and this issue still requires attention, see e.g. (Khoshbakht and Lin 2010; Sciolti et al. 2012; Ghiassi et al. 2013a).

The available information in the literature shows that the bond behavior may undergo degradation due to environmental actions that can affect the structure performance or even cause FRP premature debonding. The bond degradation due to environmental actions can be investigated by conducting accelerated ageing tests usually performed by exposing the specimens to a harsh environment and monitoring the changes in the bond behavior with exposure time. Hygrothermal conditions (combined effect of temperature and moisture), being more similar to 
real exposure conditions, are known to affect the degradation process (Cromwell et al. 2011).

Moisture exposure plays an important role in the durability of bond and leads to a reduction of the bond strength and fracture energy (Ouyang and Wan 2008; Lau and Buyukozturk 2010; Sciolti et al. 2012). The failure mode often changes from cohesive to adhesive, the debonding force decreases and the bond force-slip diagrams become non-linear. These observations are usually attributed to extensive moisture plasticization of the polymer adhesive and additional breakage of interfacial bonds (Wan et al. 2006). Temperature cycles below the epoxy glass transition temperature may cause degradation in the bond due to the imposed thermal fatigue and thermal incompatibility between FRP and the substrate (Karbhari et al. 2003). Furthermore, exposure to subzero temperatures and freezethaw cycles cause degradation in the bond behavior. Still, the combined effect of temperature cycles and moisture, the so-called hygrothermal ageing, is still not well known.

This paper presents the results of an experimental investigation devoted to the hygrothermal durability of the bond in FRP-strengthened bricks. Accelerated ageing tests were performed on FRP-strengthened brick elements and the degradation of the bond due to hygrothermal conditions was investigated. Two different hygrothermal conditions were considered aiming at understanding the coupled effect of temperature cycles and relative humidity. The specimens were prepared by application of GFRP composites on solid brick specimens following the wet lay-up procedure. The degradation of bond was quantitatively assessed by performing conventional single-lap shear bond tests. The changes in mechanical properties of the material constituents have also been investigated. Finally, the results are presented and critically discussed. 


\section{Experimental program}

The experimental program addresses an investigation on the degradation of bond behavior in FRP-strengthened masonry due to hygrothermal conditions. The material characterization tests, specimens' preparation, accelerated exposure conditions and post-ageing test methods are presented in this section.

\subsection{Material characterization}

Mechanical tests have been performed on brick, epoxy resin, and GFRP coupon specimens to investigate the effect of environmental exposures on the properties of material constituents, see Fig. 1. The tests are performed after different periods of exposure. The mechanical properties of the unconditioned specimens are also evaluated to provide a baseline for the results obtained from the conditioned specimens. The tests have been performed according to the relevant test standards and the results are presented as the mean value of five specimens.

\subsubsection{Brick}

The mechanical properties of bricks were obtained according to standards (EN 772-1 2002) and (UNI EN 8942-3 1986) in terms of compressive strength, $f_{\mathrm{cb}}$ and flexural tensile strength, $f_{\mathrm{tb}}$. The compressive strength was obtained by performing compressive tests on $40 \mathrm{~mm}$ height brick cubes, in the flatwise direction (the direction normal to the largest surface of the brick). Three point bending tests were performed on $160 \times 40 \times 40 \mathrm{~mm}^{3}$ brick specimens to obtain the flexural tensile strength. Both tests were carried out with an Instron testing machine at a displacement rate of $5 \mu \mathrm{m} / \mathrm{sec}$. 


\subsubsection{Epoxy resin and primer}

Mechanical properties of the epoxy resin and primer (tensile strength and elastic modulus) were determined from tests on dog-bone shape specimens, see Fig. 1(c). Although seven days are proposed for curing the epoxy resin in the technical datasheet provided by the manufacturer, the specimens were previously cured for 60 days at room temperature. Previous studies have shown that high curing times are necessary for complete curing of cold-cured epoxy resins (Frigione et al. 2006a; Sciolti et al. 2012).

The specimens' preparation and tensile tests are conducted following the (ISO 527-1 2012). The tests were carried out with an Instron testing machine at a displacement rate of $0.01 \mathrm{~mm} / \mathrm{min}$. Deformation of the specimens was monitored by a clip gauge placed on the middle of the specimens.

The glass transition temperature $\left(T_{\mathrm{g}}\right)$ of the epoxy resin was obtained by means of the DSC (Differential Scanning Calorimetry) method. The thermal scans were carried out between $5^{\circ} \mathrm{C}$ and $200^{\circ} \mathrm{C}$ with a heating rate of $10^{\circ} \mathrm{C} / \mathrm{min}$. The $T_{\mathrm{g}}$ was calculated as the mean value of four tests.

\subsubsection{GFRP composite}

Regarding the composite materials, the specimens preparation and mechanical tests were conducted according to (ISO 527-1 2012). The mechanical properties are obtained in terms of tensile strength, $f_{\mathrm{tf}}$, and elastic modulus, $E_{\mathrm{f}}$. The GFRP coupons were prepared following the wet lay-up procedure according to the code specifications, Fig. 1(d). Throughout this study, changes in the tensile strength and elastic modulus of GFRP coupons are normalized to the specimens' thickness in accordance with (ASTM D7565-10 2010). In wet lay-up procedures the specimens' thickness varies and the normalization of the mechanical properties by 
the thickness can provide an accurate baseline for comparison (Cromwell et al. 2011).

\subsubsection{Mechanical properties of unconditioned specimens}

The mechanical properties of the unconditioned material constituents are presented in Table 1, in terms of mean value and coefficient of variation $(\mathrm{CoV})$. The tests are performed as explained in the last sections. It can be seen that the brick presents low CoVs (about 4\%). The GFRP coupons exhibit high CoVs (about 15\%) due to the manufacture process, whereas the epoxy resin and primer exhibit lower CoVs (about 10\%).

\subsection{Specimens' preparation}

The GFRP sheets were applied to the brick surface following the wet lay-up procedure. The bricks were dried in the oven for around $24 \mathrm{hr}$ at $100{ }^{\circ} \mathrm{C}$ before application of the GFRP sheets. After 24 hours, the changes in the weight of bricks were monitored each hour. The brick were assumed to be in the dry state when their weight was constant in two consecutive weight measurements. After cleaning the brick surface, a two-part primer was applied to the brick's surface for preparation of the substrate surface before GFRP application. Finally, a two-part epoxy resin was used as the matrix of the composite material and also for adhesion to the masonry substrate. The $50 \mathrm{~mm}$ width GFRP sheets were applied on the brick's surface along $150 \mathrm{~mm}$ length of the brick with a $40 \mathrm{~mm}$ unbounded part at the loaded end, see Fig. 2.

\subsection{Environmental exposure}

The experimental program consisted of exposing the specimens to two different hygrothermal conditions in a climatic chamber. The aim was to investigate the 
degrading effect of hygrothermal conditions on the bond behavior. The details of exposure conditions are shown in Fig. 3.

In the first exposure, exposure HT, the specimens were exposed to 6 hours temperature cycles from $+10{ }^{\circ} \mathrm{C}$ to $+50{ }^{\circ} \mathrm{C}$ and constant relative humidity of $90 \%$. In each cycle, the temperature was kept constant at $+10^{\circ} \mathrm{C}$ for 2 hours, subsequently increased to $+50^{\circ} \mathrm{C}$ in 1 hour, followed by 2 hours constant temperature at $+50^{\circ} \mathrm{C}$. Then, the temperature was decreased again to $+10^{\circ} \mathrm{C}$ in 1 hour resulting in 6 hours cycles of exposure. The $+50^{\circ} \mathrm{C}$ is relatively a high temperature chosen to accelerate the degradation phenomenon while being far enough from the epoxy resin $T_{\mathrm{g}}\left(70^{\circ} \mathrm{C}\right)$. Since environmental conditions can cause reduction of $T_{\mathrm{g}}$ in epoxy resins, the maximum temperature in the thermal cycles should avoid reaching the $T_{\mathrm{g}}$ of the epoxy resin during the tests (Karbhari 2007). The specimens were subjected to a total of 225 cycles of this exposure.

In the second exposure, exposure FT, the specimens were exposed to temperature cycles from $-10{ }^{\circ} \mathrm{C}$ to $+30{ }^{\circ} \mathrm{C}$ and, again, constant relative humidity of $90 \%$. The aim was to investigate the effect of freeze-thaw conditions on the bond behavior while having the minimum number of changes comparing to the first exposure. Therefore, a similar rate of exposure was used with $20^{\circ} \mathrm{C}$ decrement of the maximum and minimum temperatures. The specimens were subjected to a total of 300 cycles during this exposure.

\subsection{Post-ageing tests}

Post-ageing tests were performed after each two weeks of environmental exposure (around each 60 cycles), both for mechanical characterization of materials and bond behavior. The specimens were taken from the climatic chamber after stabilizing at $+500^{\circ} \mathrm{C}$ in $\mathrm{HT}$ cycles and $+30^{\circ} \mathrm{C}$ in FT cycles. The specimens were 
then stabilized in laboratory conditions for four days before performing the postageing tests.

The post-ageing tests can be categorized as mechanical characterization of materials and bond assessment tests. Five specimens were tested in each exposure period and the average results are presented next.

\subsubsection{Degradation of material properties}

The changes in mechanical properties of materials were investigated by performing compressive tests on brick samples and tensile tests on epoxy resin and GFRP coupon specimens.

\subsubsection{Degradation of bond properties}

Bond assessment tests consisted of two phases. In the first phase, the specimens were inspected visually and photos were taken after each period of the exposure to investigate the existence of visible damages or delaminations in the interfacial region. In the second phase, the degradation of the bond behavior was investigated quantitatively by performing conventional single-lap shear bond tests.

The bond tests were performed using a closed-loop servo-controlled testing machine with a maximum load capacity of $50 \mathrm{kN}$. A rigid steel frame was used to support the specimens appropriately and avoid misalignments in the load application. The specimens were placed on the steel frame and firmly clamped to it as shown in Fig. 4(a). The tests were driven under displacement control conditions with reference to the LVDT sensor placed at the loaded end of the FRP composite. The specimens were pulled monotonically at a rate of $5 \mu \mathrm{m} / \mathrm{sec}$. The resulting force, F, was measured by means of a load cell. The relative slip between the GFRP and the brick was measured with the LVDTs placed along the bonded length, see Fig. 4(b). 


\section{Results and discussion}

\subsection{Degradation of material properties}

\subsubsection{Brick}

The changes in the compressive strength of bricks in the two hygrothermal exposures are shown in Fig. 5. The changes in the bricks' compressive strength are negligible in both exposure conditions with a low CoV (maximum 10\%). This results show the good resistance of the bricks to the environmental exposures considered in this study.

\subsubsection{Epoxy resin}

The changes in the mechanical properties of epoxy resin, namely elastic modulus and tensile strength, are presented in Fig. 6. The elastic modulus has negligible changes in both exposure types, see Fig. 6(a, c). However, some degradation seems to occur in the tensile strength of the epoxy resin. In exposure HT, a total reduction of $20 \%$ reduction of tensile strength was observed after 225 cycles of exposure. In exposure FT, the epoxy resin showed a better durability performance with $8 \%$ reduction of tensile strength after 300 cycles of exposure. Longer cycles of exposure are needed for a clear conclusion. The CoVs of the tests in both exposures were in the range of $2 \%$ to $13 \%$ which seem reasonable for testing material properties (Haldar and Mahadevan 2000).

The observed reduction in mechanical properties can be due to different degradation mechanisms. Moisture exposure causes plasticization and hydrolysis of epoxy resin (Moy and Karasz 1980; Carol and Schutte 1994; Lau and Buyukozturk 2010). The water absorbed by the epoxy resin acts as a plasticizer, and the result of plasticization is usually reduction of $T_{\mathrm{g}}$ and mechanical properties of epoxy resin (Frigione et al. 2006b). For long exposure periods, the 
possibility of resin degradation increases while hydrolytic reactions or chain scission may also occur, which can strongly affect the resin performance (Frigione et al. 2006b). The possible observed increase in the degradation rate after 150 cycles of HT exposure can be due to this fact, see Fig. 6(b). On the other hand, exposure to high temperatures below the epoxy $T_{\mathrm{g}}$ results in an increment of moisture diffusion coefficient and epoxy post-curing (Carol and Schutte 1994). While the former lead to higher moisture uptake and consequently higher mechanical degradation, the latter can cause an improvement in the mechanical properties.

\subsubsection{GFRP composite}

The changes in mechanical properties of GFRP coupons are presented in Fig. 7. Both hygrothermal exposures imposed some degradation of mechanical properties in GFRP coupons. However, more degradation was observed in the specimens exposed to HT conditions. The elastic modulus and tensile strength of GFRP coupons decreased $23 \%$ and $22 \%$, respectively, after 225 cycles of HT exposure. On the other hand, $18 \%$ and $10 \%$ reduction of elastic modulus and tensile strength, respectively, was observed in the specimens after 300 cycles of FT conditions. The CoVs of the experimental results in both exposures were in the range of $5 \%$ to $12 \%$ which are again typical for testing material properties (Haldar and Mahadevan 2000).

The observed degradation can be attributed to different degrading mechanisms. GFRP coupons absorb moisture when exposed to moisture conditions. Moisture causes degradation in the epoxy resin properties as it was described in the last section. Moreover, the water attacks glass fibers resulting in degradation of their mechanical properties and surface energy. The fiber/epoxy interface may also 
degrade due to the degradation of fiber and epoxy resin and also the produced osmotic pressure at the interface (Karbhari 2007). On the other hand, the cyclic temperature can cause micro-cracking due to difference between thermal expansion coefficients of glass fibers and epoxy resin (Dutta and Hui 1996; Karbhari 2002). Meanwhile, the $50{ }^{\circ} \mathrm{C}$ temperature in HT cycles may cause postcuring of epoxy resin and increment of moisture absorption coefficient, as mentioned previously.

\subsection{Degradation of bond behavior}

The changes in the bond behavior due to environmental conditions have been investigated by visual inspection of the specimens (qualitatively) and by singlelap shear bond tests (quantitatively). The obtained results are presented and discussed in this section.

\subsubsection{Visual inspection}

All specimens have been visually inspected after each two weeks of exposure, before performing the debonding tests, for investigating the existence of visible interfacial damage or FRP delaminations. Progressive FRP delaminations were observed in some specimens with the increase in the exposure cycles, see Fig. 8. The delaminations, being at the FRP/brick interface, were generally larger in specimens subjected to HT cycles. Fig. 8 presents reduction of the bonded area of the specimens with exposure time normalized by the initial bonded area. The values are presented as the average of the five inspected specimens. One representative specimen in each exposure period is also shown in this graph. The transparent nature of the epoxy resin allowed detection of the debonded areas in the specimens by visual inspection. However, for better detection and precise quantification of the delamination sizes IR thermography technique was used. The 
detailed description of the technique and the obtained results can be found in Ghiassi et al (2014).

The observed delaminations in the specimens can be attributed to the thermal incompatibility between the composite material and the brick used in this study. The thermal expansion coefficient of clay bricks is in order of $5 \times 10^{-6} \% \mathrm{C}$ (Kralj et al. 1991). The thermal expansion coefficient of E-glass fibers is similar to the one from the bricks, while for epoxy resin it is in the range of $30 \sim 54 \times 10^{-6} / \mathrm{C}$ (CNRDT 200 2004). This large difference of thermal expansion coefficient, between epoxy resin and glass fibers / bricks, produces interfacial thermal strains at the fiber/epoxy and brick/epoxy interfaces. Cyclic temperature conditions amplify this effect by producing thermal fatigue and may cause FRP delaminations from the brick surface during the environmental exposures. On the other hand, it has been observed that the thermal expansion coefficient of epoxy resins is much lower at low and negative temperatures (Dohnalek 2006). Therefore, the interfacial strains produced due to thermal incompatibility are lower for the freeze-thaw conditions. This explains the smaller delaminated areas observed in the specimens exposed to the FT conditions in this study.

\subsubsection{Single-lap shear bond tests}

Fig. 9 shows the effect of two hygrothermal exposures on the debonding force. It can be observed that the debonding force has been progressively decreased with the number of exposure cycles. The debonding force decreased $17 \%$ and $45 \%$ after 120 and 225 cycles of HT exposure, respectively. Meanwhile, it decreased $4 \%, 6 \%$, and $14 \%$ after 120,225 , and 300 cycles of FT exposure, respectively. A comparison between the obtained results shows that the HT exposure induces higher degradation on the specimens used in this study, see Fig. 10. 
Moreover, the debonding process during the tests changed from a brittle to a progressive and less brittle mode. Similar changes in the bond behavior have also been reported in the literature for the specimens exposed to freeze-thaw and wetdry cycles, see e.g. (Davalos et al. 2008).

The bond fracture energy in each exposure period can be obtained according to (CNR-DT 200 2004) as follows:

$G_{f}=\frac{P_{\max }^{2}}{b_{f}^{2}\left(2 E_{f} t_{f}\right)}$

where, $P_{\max }$ is the debonding force, $b_{f}$ is the FRP width, $E_{f}$ is the FRP elastic modulus, and $t_{f}$ is the FRP thickness.

The average bond fracture energy value reaches $0.38 \mathrm{~N} / \mathrm{mm}$ for the reference specimens. Changes in the fracture energy of the specimens due to hygrothermal conditions are shown in Fig. 11. The fracture energy has negligible changes due to FT conditions, while a large degradation is observed in the specimens exposed to HT conditions. The interfacial fracture energy has decreased $8 \%$ and $60 \%$ after exposure to 120 and 225 cycles of HT exposure, respectively. Meanwhile, no conclusion can be made for degradation in FT exposure.

Assuming that the FRP width and thickness are constant during the hygrothermal exposures, the debonding force is directly related to the square root of interfacial fracture energy and FRP elastic modulus, see Eq (1). Therefore, the participation of each factor in degradation of debonding force can be obtained by plotting the changes in the normalized square root of fracture energy and FRP elastic modulus as shown in Fig. 12.

It can be seen that in exposure HT, Fig. 12(a), the interfacial degradation of the bond has a major effect on the global bond degradation comparing to the FRP elastic modulus. The interfacial degradation can be attributed to the existing 
thermal incompatibility inside the composite system and FRP-brick interface. Moreover, the presence of moisture in the interface causes degradation of the bond strength and fracture energy (Ouyang and Wan 2008; Lau and Buyukozturk 2010; Sciolti et al. 2012). However, isolating the degradation due to thermal incompatibility problem and moisture presence is a complex task which needs an extensive experimental program.

In exposure FT, Fig. 12(b), the effect of FRP mechanical properties and interfacial behavior is similar in the observed degradation. The presence of water at the interface region is an important factor in degradation due to the freeze-thaw exposure which is directly related to the porosity of superficial layer of the bricks (Colombi et al. 2010). Since the surface layer of the bricks used in this study has a low porosity, the observed performance of the system against freeze-thaw conditions was expected. Moreover, as explained before, since the thermal expansion coefficient of epoxy resins decreases at low temperature, less thermal incompatibility problem is expected in this exposure condition.

Regarding the failure mode, three different failures were observed in the specimens: (1) cohesive failure within a thick layer of brick; (2) cohesive failure within a superficial layer of brick, next denoted by interfacial cohesive; (3) adhesive failure at the FRP/brick interface. For both hygrothermal exposures, a progressive change of failure mode from cohesive to adhesive was observed in the specimens with the increase of exposure cycles, Fig. 13. Such a change in the failure mode of the specimens, also reported in (Green et al. 2000), can be attributed to the observed bond degradation during environmental exposures. The contribution of each failure mode in the debonding behavior is presented in Fig. 14. These values are obtained by measuring the area corresponding to each failure mode on the failure surface of the specimens after debonding tests. Then, the 
average values are obtained from the specimens belonging to each exposure period. It can be observed that the contribution of the cohesive failure reduces along the time. Meanwhile, adhesive failure governs the behavior with the increase of the exposure cycles.

\section{Conclusions}

The results of an extensive experimental program aimed at investigating the durability of FRP-masonry systems were presented in this study. Accelerated ageing tests were performed following two different hygrothermal conditions consisting of thermal cycles from $+10^{\circ} \mathrm{C}$ to $+50{ }^{\circ} \mathrm{C}$ (90\% R.H.) called HT exposure, and $-10{ }^{\circ} \mathrm{C}$ to $+30{ }^{\circ} \mathrm{C}(90 \%$ R.H.) called FT exposure. The bond degradation was studied by visual inspection and single-lap shear bond tests. The changes in mechanical properties of material constituents were also investigated. Based on the obtained results, the following conclusions can be drawn:

- The hygrothermal exposures did not affect the mechanical properties of bricks. However, epoxy resin and GFRP coupons showed some degradation. Generally, higher degradation levels were observed due to exposing the specimens to HT conditions. Elastic modulus of epoxy resin did not change significantly in both exposure tests. On the other hand, $20 \%$ and $10 \%$ decrease in tensile strength were observed after 225 cycles of exposure to HT and FT exposures, respectively. In case of GFRP coupons, Elastic modulus and tensile strength slightly decreased being more severe in HT cycles.

- FRP delamination was observed at the FRP/brick interface after exposure to environmental conditions. The detached areas increased with the number of cycles. The detachments were larger in specimens exposed to HT conditions. 
- A progressive degradation of bond strength and fracture energy was observed in the specimens for both exposure types. The observed degradation was more severe in the specimens subjected to HT cycles.

- In both exposure types, the failure mode changed progressively from cohesive failure in the brick to adhesive failure at the FRP-brick interface with exposure time.

\section{Acknowledgements}

This work was developed within the framework of the RILEM Technical Committee "223-MSC: Masonry Strengthening with Composite Materials". The financial support from the project FP7ENV-2009-1-244123-NIKER of the 7th Framework Program of the European Commission is gratefully acknowledged. The first author also acknowledges the financial support of the Portuguese Science Foundation (Fundação de Ciência e Tecnologia, FCT), through grant SFRH/BD/80697/2011.

\section{References}

ASTM D7565-10 (2010) Standard test method for determining tensile properties of fiber reinforced polymer matrix composites used for strengthening of civil structures., vol ASTM D7565.

Capozucca R (2012) Effects of mortar layers in the delamination of GFRP bonded to historic masonry. Compos Part B 44 (1):639-49.

Carol L, Schutte CL (1994) Environmental durability of glass-fiber composites. Mater Sci Eng A 13 (7):265-323.

CNR-DT 200 (2004) Guide for the design and construction of externally bonded FRP systems for strengthening existing structures. CNR-DT 200/2004. National Research Council, Rome.

Colombi P, Fava G, Poggi C (2010) Bond strength of CFRP-concrete elements under freeze-thaw cycles. Compos struct 92 (4):973-83.

Cromwell JR, Harries KA, Shahrooz BM (2011) Environmental durability of externally bonded FRP materials intended for repair of concrete structures. Constr Build Mater 25 (5):2525-39.

Davalos JF, Kodkani SS, Ray I, Lin C (2008) Fracture evaluation of GFRPconcrete interfaces for freeze-thaw and wet-dry cycling. J Compos Mater 42 (14):1439-66.

Dohnalek P (2006) Environmental durability of FRP bond to concrete subjected to freeze-thaw action., MSc Thesis, MIT.

Dutta PK, Hui D (1996) Low-temperature and freeze-thaw durability of thick composites. Compos Part B 27 (3-4):371-9

EN 772-1 (2002) Methods of test for masonry units -Part 1: Determination of compressive strength. 
Faella C, Camorani G, Martinelli E, Paciello S, Perri F (2012) Bond behavior of FRP strip glued on masonry: Experimental investigation and empirical formulaiton. Constr Build Mater 31:353-63.

Frigione M, Aiello MA, Naddeo C (2006a) Water effects on the bond strength of concrete/concrete adhesive joints. Constr Build Mater 20 (10):957-70.

Frigione M, Lettieri M, Mecchi AM (2006b) Environmental effects on epoxy adhesives employed for restoration of historical buildings. J Mater Civil Eng 18 (5):715-22.

Ghiassi B, Marcari G, Oliveira DV, Lourenço PB (2012a) Numerical analysis of bond behavior between masonry bricks and composite materials. Eng Struct 43:210-20.

Ghiassi B, Oliveira DV, Lourenço PB, Marcari A (2012b) Numerical study of the role of mortar joints in the bond behavior of FRP-strengthened masonry. Compos Part B 46:21-30.

Ghiassi B, Marcari G, Oliveira DV, Lourenço PB (2013a) Water degrading effects on the bond behavior in FRP-strengthened masonry. Compos. Part B, 54, 11-19.

Ghiassi B, Silva SM, Oliveira DV, Lourenço PB, Bragança L (2014). Assessment of the bond quality degradation in FRP-strengthened masonry using IR thermography technique. J Nondestruct Eval, doi: 10.1007/s10921-0140238-8.

Grande E, Imbimbo M, Sacco E Experimental investigation on the bond behavior of CFRP laminates glued on clay bricks. In: CICE2008, Zurich, Switzerland, 2008.

Green MF, Bibsy LA, Beaudoin Y, Labossière P (2000) Effect of freeze-thaw cycles on the bond durability between fiber reinforced polymer plate reinforcement and concrete. Can J Civil Eng 27 (5):949-59.

Haldar A, Mahadevan S (2000) Probability, reliability and statistical methods in engineering design. John Wiley.

Hollaway LC (2010) A review of the present and future utilisation of FRP composites in the civil infrastructure with reference to their important inservice properties. Constr Build Mater 24 (12):2419-45.

ISO 527-1 (2012) Plastics-determination of tensile properties- Part 1: general principles.

Karbhari VM (2002) Reponse of fiber reinforced polymer confined concrete exposed to freeze and freeze-thaw regimes. J Compos Constr 6 (1):35-40.

Karbhari VM (2007) Durability of composites for civil structural applications. CRC Press, Washington, DC.

Karbhari VM, Chin W, Hunston D, Benmokrane B, Juska T, Morgan R, Lesko JJ, Sorathia U, Reynaud D (2003) Durability gap analysis for fiber-reinforced polymer composites in civil infrastructures. J Compos Constr 7 (3):23847.

Khoshbakht M, Lin MW (2010) A finite element model for hygro-thermomechanical analysis of masonry walls with FRP reinforcement. Finite Elem Anal Des 46 (10):783-91.

Kralj B, Pande GN, Middleton J (1991) On the mechanics of frost damage to brick masonry. Compos Struct 41 (1):53-66.

Lau D, Buyukozturk O (2010) Fracture characterization of concrete/epoxy interface affected by moisture. Mech Mater 42 (12):1031-42.

Moy P, Karasz F (1980) Epoxy-water interactions. Polym Eng Sci 20 (4):315-19. 
Ouyang Z, Wan B (2008) Experimental and numerical study of water effect on bond fracture energy between FRP and concrete in moist environments. J Reinf Plast Compos 27 (2):205-23.

Sciolti MS, Aiello MA, Frigione M (2012) Influence of water on bond behavior between CFRP sheet and natural calcareous stones. Compos Part B 43 (8):3239-50.

UNI EN 8942-3 (1986) Clay bricks and blocks. Test methods.

Valluzzi MR, Oliveira DV, Caratelli A, et al (2012) Round robin test for composite-to-brick shear bond characterization. J Mater Struct 45(12):1761-91

Wan B, Petrou MF, Harries KA (2006) Effect of the presence of water on the durability of bond between CFRP and concrete. J Reinf Plast Compos 25 (8):875-90. 


\section{List of Tables}

Table 1 Material properties

\section{List of Figures}

Fig. 1 Tests on material components (dimensions in mm): (a) brick compressive specimen; (b) brick flexural tensile specimen; (c) epoxy resin and primer tensile specimen; (d) GFRP coupon tensile specimen

Fig. 2 Specimens' details for single-lap shear bond tests

Fig. 3 Hygrothermal cycles adopted

Fig. 4 Single-lap shear bond tests: (a) test setup (perspective); (b) specimens instrumentation (front view)

Fig. 5 Effect of hygrothermal exposures on the compressive strength of bricks: (a) exposure HT; (b) exposure FT

Fig. 6 Changes in mechanical properties of epoxy resin: (a) HT exposure-elastic modulus; (b) HT exposure-tensile strength; (c) FT exposure-elastic modulus; (d) FT exposure-tensile strength

Fig. 7 Changes in mechanical properties of GFRP: (a) HT exposure-elastic modulus; (b) HT exposure-tensile strength; (c) FT exposure-elastic modulus; (d) FT exposure-tensile strength

Fig. 8 Reduction of the bonded area due to hygrothermal exposures: (a) exposure HT; (b) exposure FT

Fig. 9 Effect of hygrothermal exposures on the debonding force: (a) exposure HT; (b) exposure FT

Fig. 10 Comparison of debonding force degradation between HT and FT exposure conditions

Fig. 11 Effect of hygrothermal exposures on the bond fracture energy

Fig. 12 Governing degrading mechanisms in the bond behavior: (a) exposure HT; (b) exposure FT

Fig. 13 Observed failure modes in shear bond tests: (a) exposure HT; (b) exposure FT

Fig. 14 Contribution of each failure mode in the debonding behavior: (a) exposure HT; (b) exposure FT 
Table 1 Material properties

\begin{tabular}{lccc}
\hline Masonry brick & & $\begin{array}{c}\text { Mean } \\
\text { value }\end{array}$ & $\mathrm{CoV}(\%)$ \\
\hline Compressive strength & $f_{\mathrm{cb}}(\mathrm{MPa})$ & 14.2 & 4.0 \\
Flexural tensile strength & $f_{\mathrm{tb}}(\mathrm{MPa})$ & 1.6 & 12.0 \\
\hline GFRP coupons & & & \\
\hline Tensile strength & $f_{\mathrm{tf}}(\mathrm{MPa})$ & 1250 & 15.0 \\
Elastic modulus & $E_{\mathrm{f}}(\mathrm{GPa})$ & 79.2 & 6.8 \\
Ultimate deformation & $\varepsilon(\%)$ & 3.0 & 20.3 \\
\hline Epoxy resin & & & \\
\hline Tensile strength & $f_{\mathrm{tm}}(\mathrm{MPa})$ & 53.8 & 9.7 \\
Elastic modulus & $E_{\mathrm{m}}(\mathrm{GPa})$ & 2.5 & 9.5 \\
Glass transition temperature & $T_{\mathrm{g}}(\mathrm{C})$ & 70 & 3.2 \\
\hline Primer & & & \\
\hline Tensile strength & $f_{\mathrm{tp}}(\mathrm{MPa})$ & 55.3 & 11.1 \\
Elastic modulus & $E_{\mathrm{p}}(\mathrm{GPa})$ & 2.9 & 6.1 \\
\hline
\end{tabular}




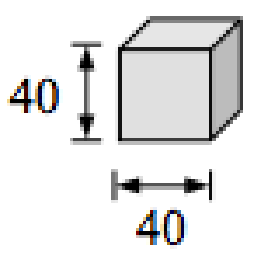

(a)

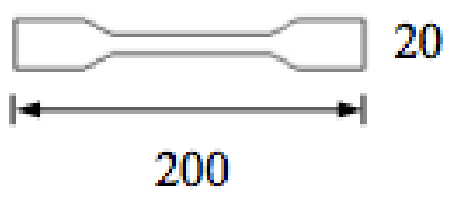

(c)

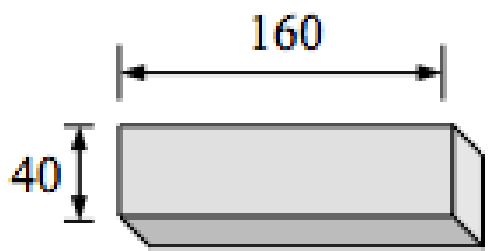

(b)

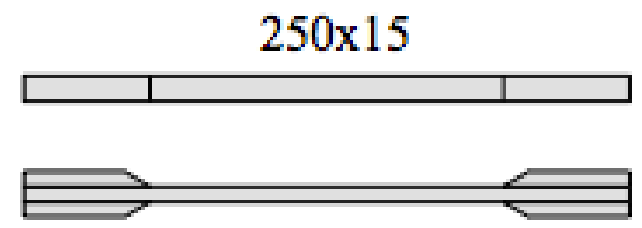

(d)

Fig. 1 Tests on material components (dimensions in $\mathrm{mm}$ ): (a) brick compressive specimen; (b) brick flexural tensile specimen; (c) epoxy resin and primer tensile specimen; (d) GFRP coupon tensile specimen 


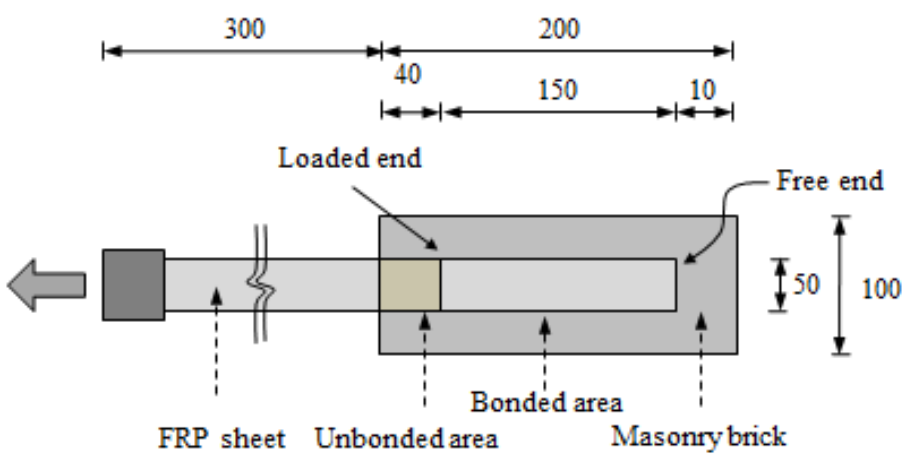

Fig. 2 Specimens' details for single-lap shear bond tests 


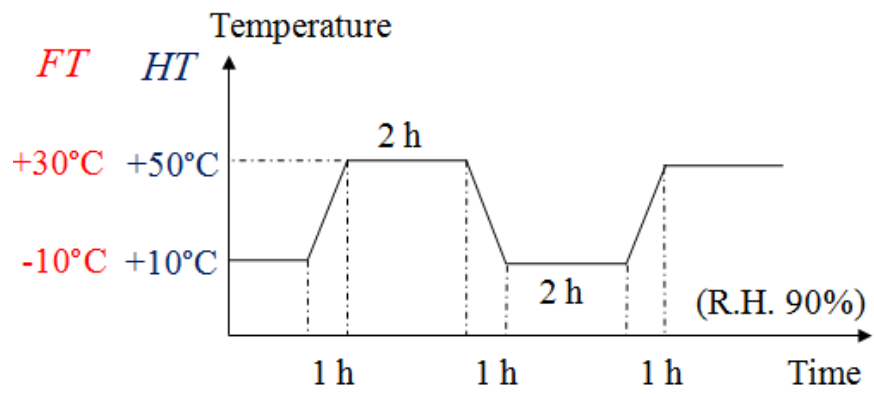

Fig. 3 Hygrothermal cycles adopted 


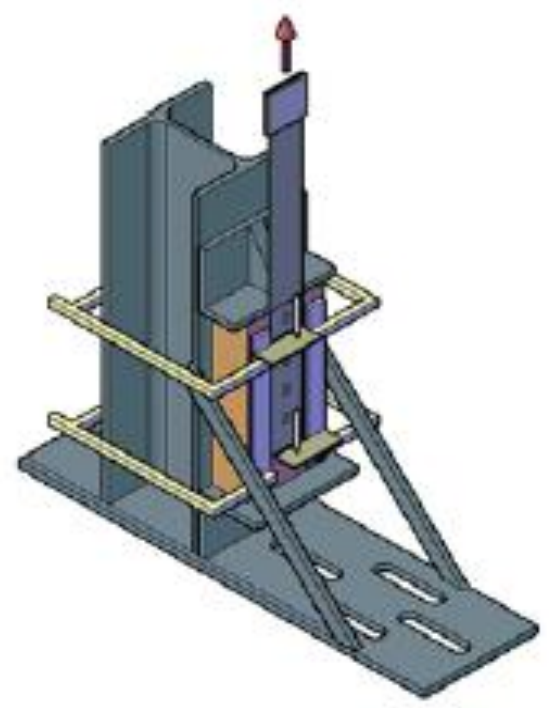

(a)

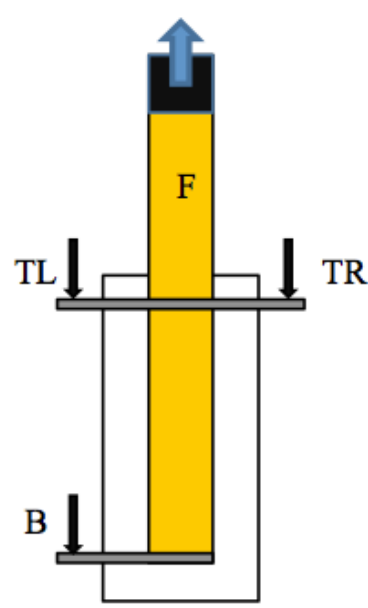

(b)

Fig. 4 Single-lap shear bond tests: (a) test setup (perspective); (b) specimens instrumentation (front view) 


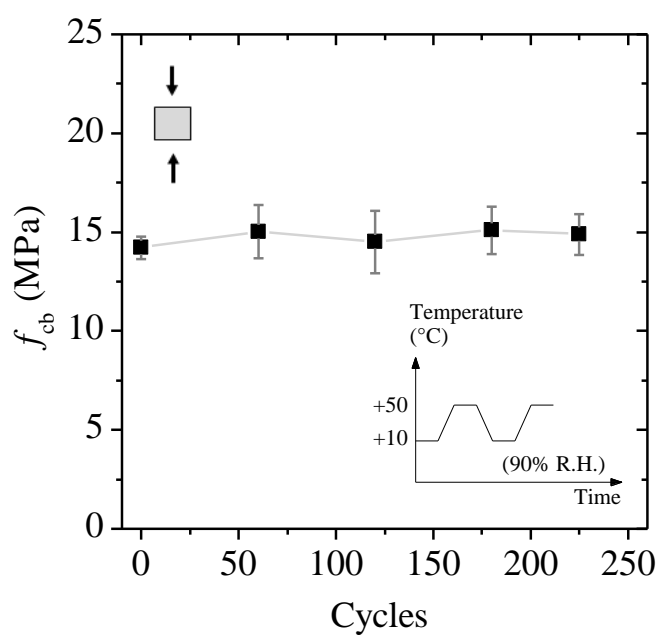

(a)

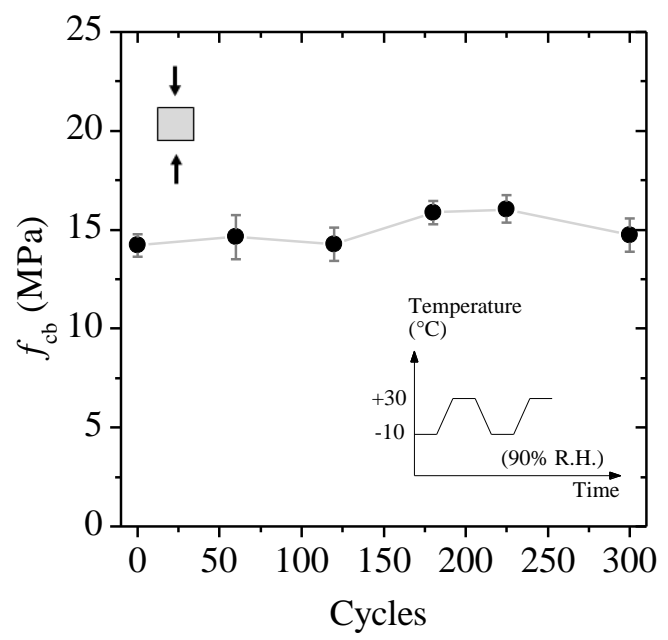

(b)

Fig. 5 Effect of hygrothermal exposures on the compressive strength of bricks: (a) exposure HT; (b) exposure FT 


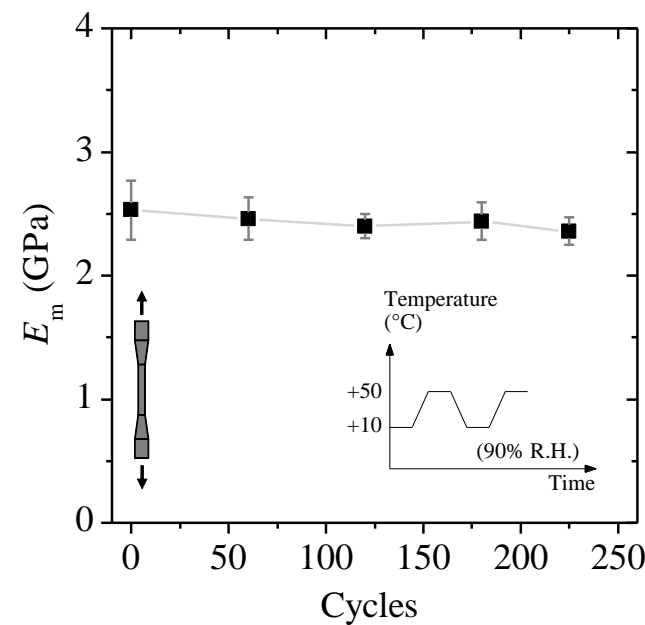

(a)

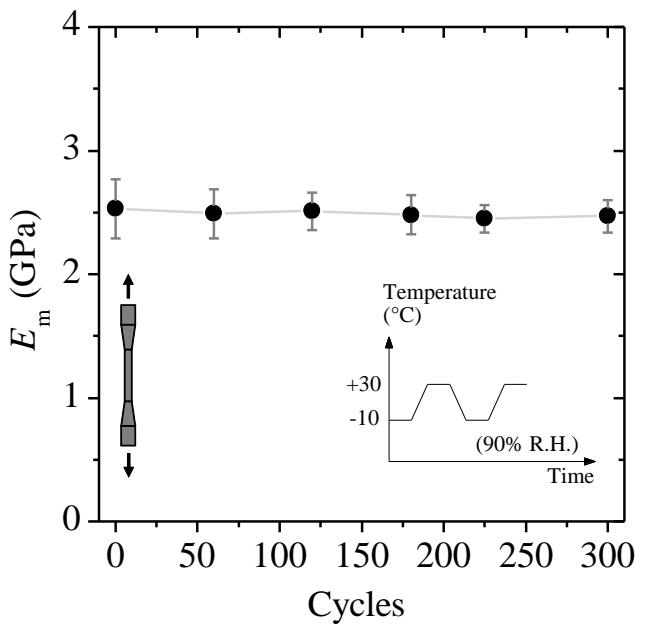

(c)

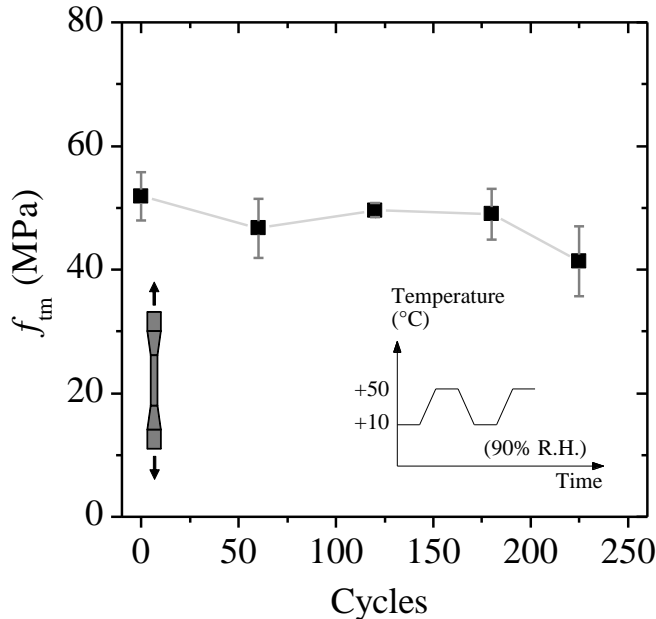

(b)

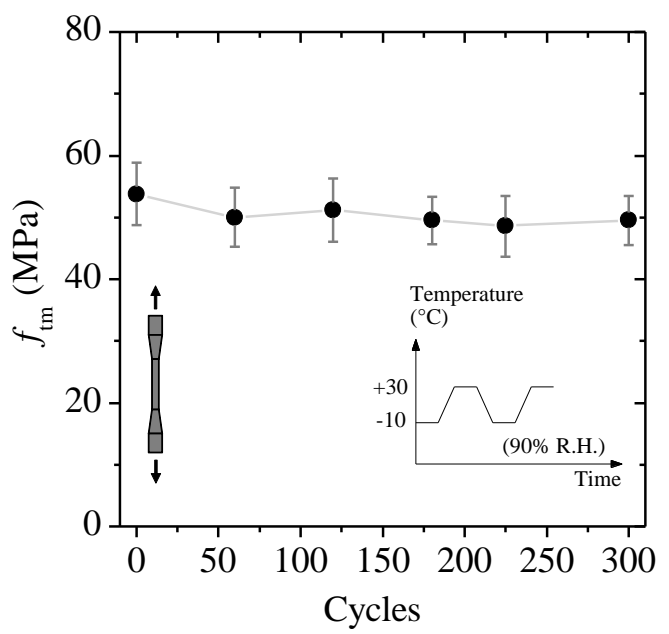

(d)

Fig. 6 Changes in mechanical properties of epoxy resin: (a) HT exposure-elastic modulus; (b) HT exposure-tensile strength; (c) FT exposure-elastic modulus; (d) FT exposure-tensile strength 


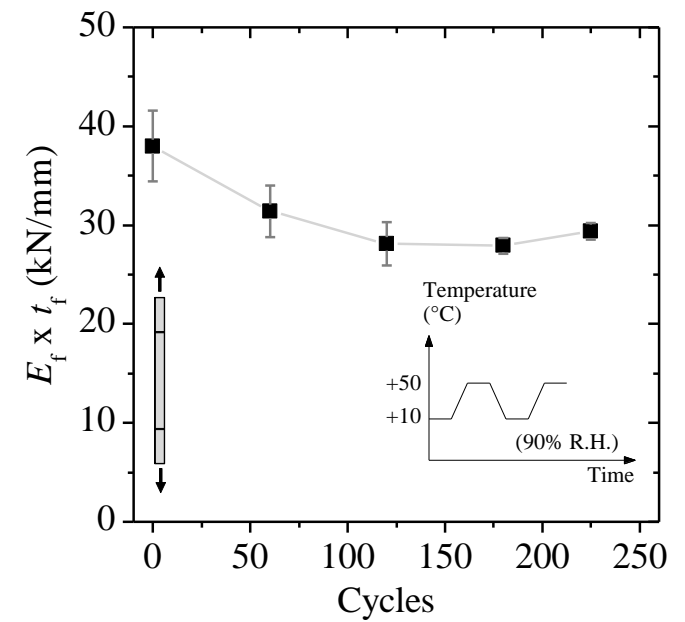

(a)

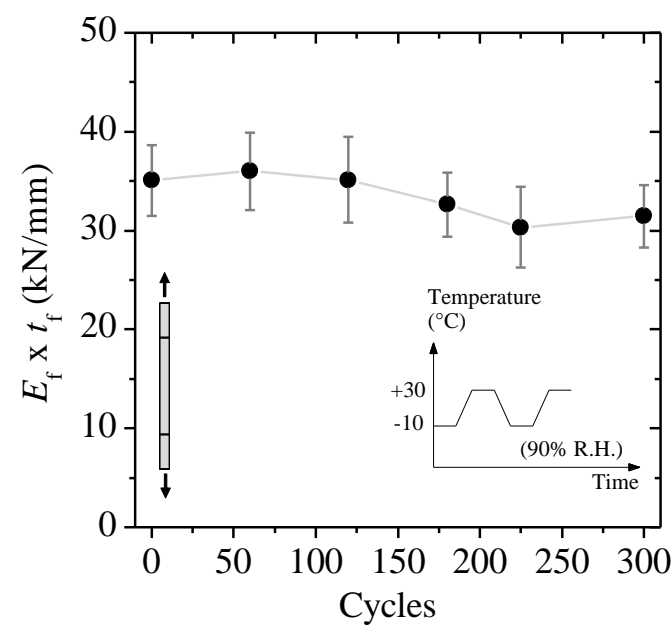

(c)

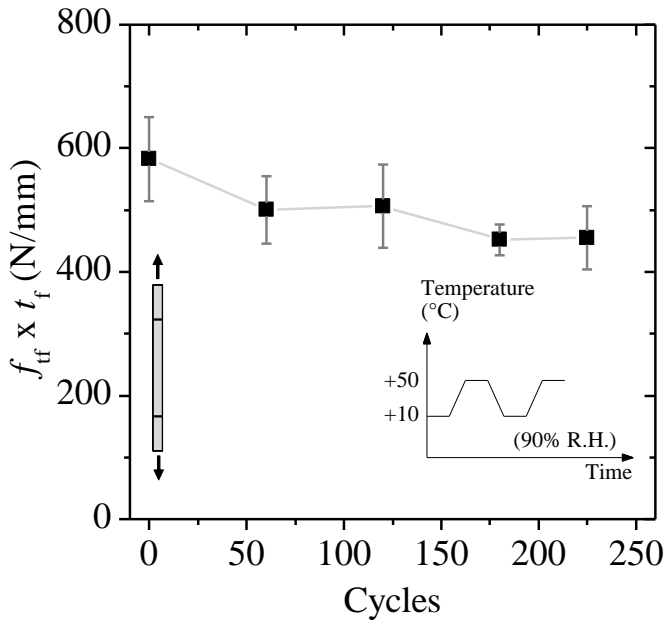

(b)

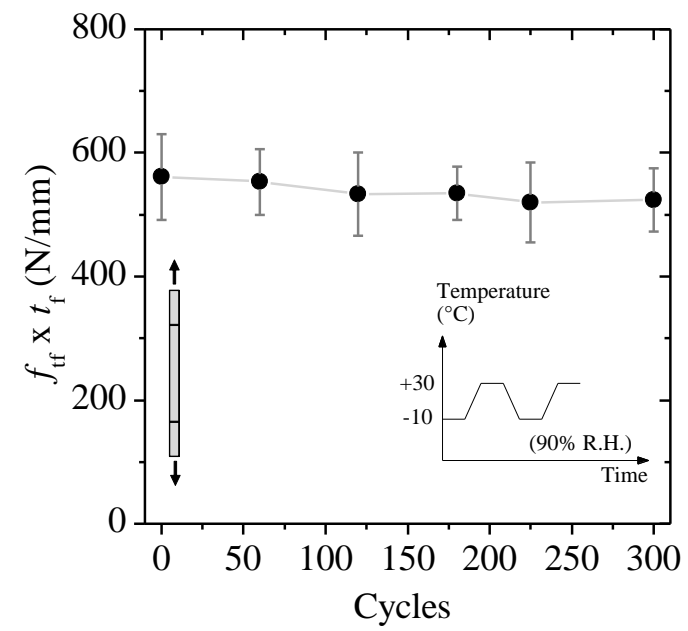

(d)

Fig. 7 Changes in mechanical properties of GFRP: (a) HT exposure-elastic modulus; (b) HT exposure-tensile strength; (c) FT exposure-elastic modulus; (d) FT exposure-tensile strength 


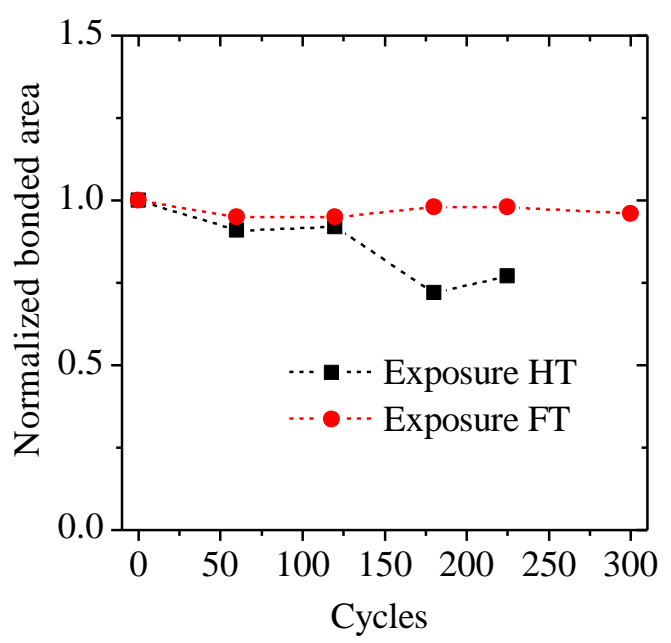

Fig. 8 Reduction of the bonded area due to hygrothermal exposures 


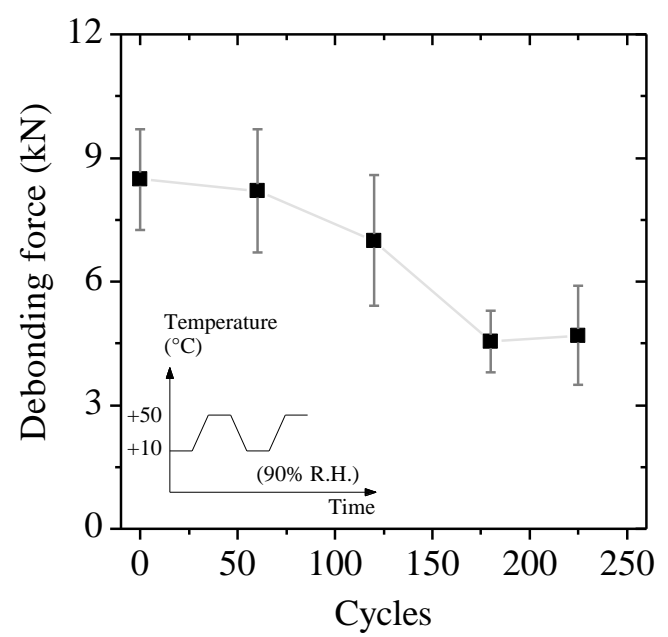

(a)

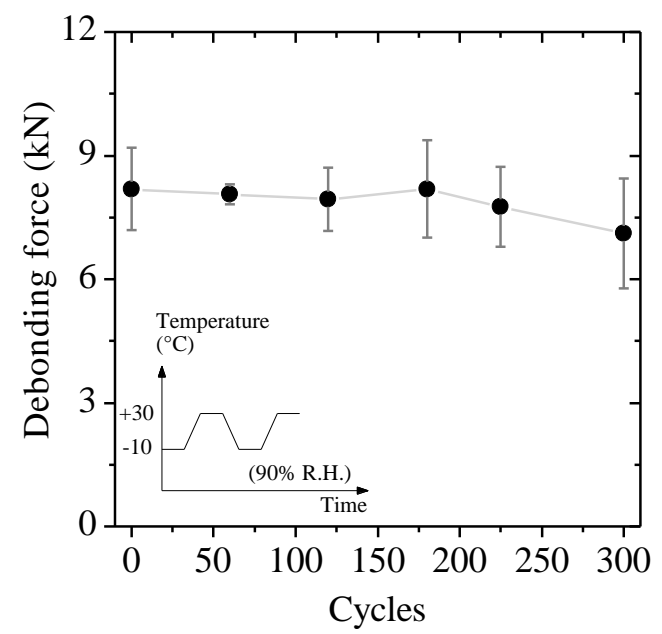

(b)

Fig. 9 Effect of hygrothermal exposures on the debonding force: (a) exposure HT; (b) exposure FT 


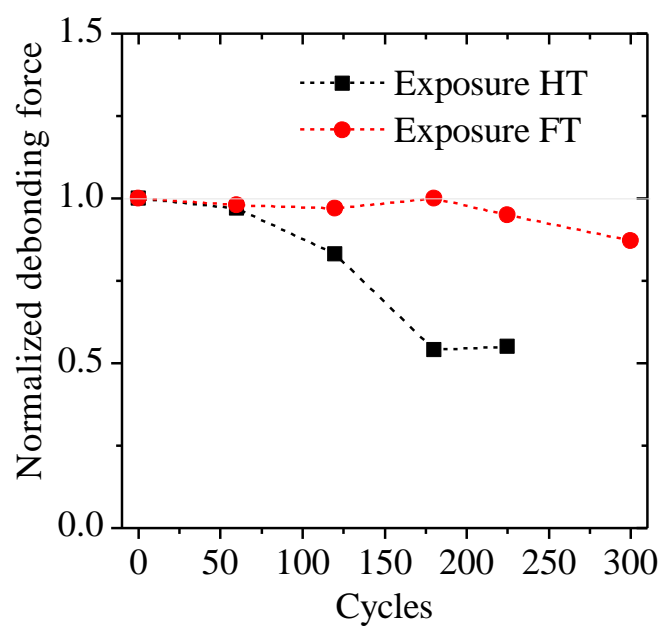

Fig. 10 Comparison of debonding force degradation between HT and FT exposure conditions 


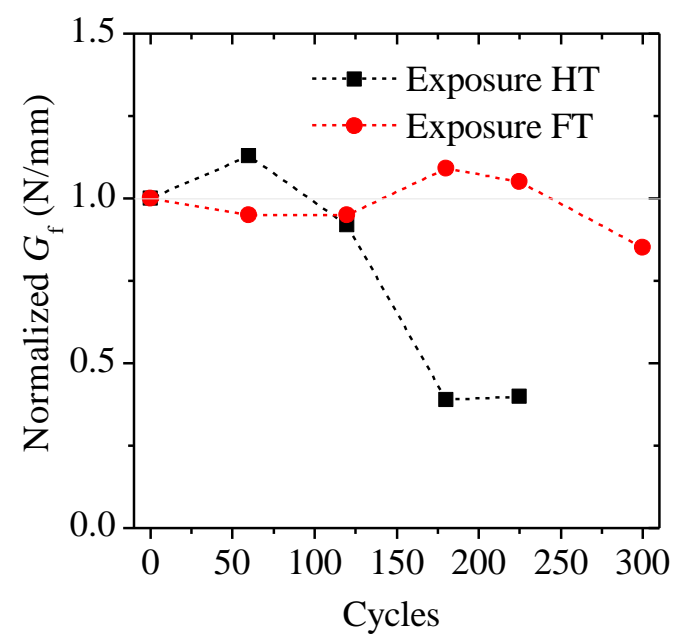

Fig. 11 Effect of hygrothermal exposures on the bond fracture energy 


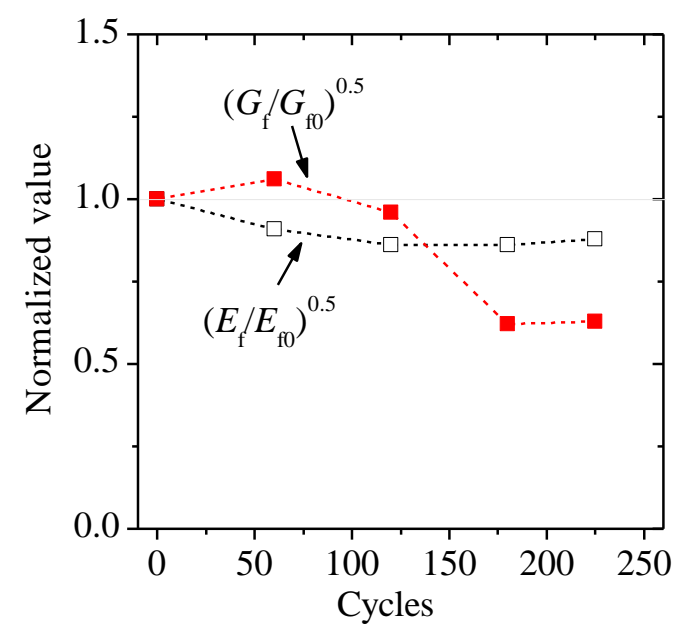

(a)

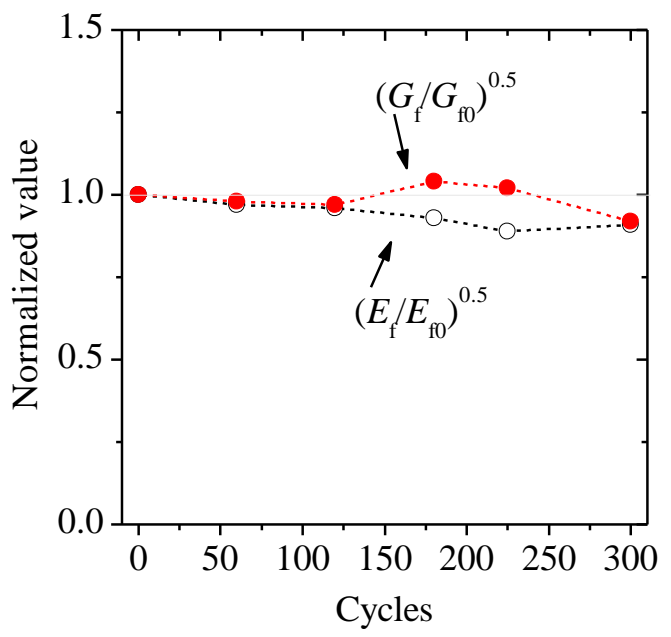

(b)

Fig. 12 Governing degrading mechanisms in the bond behavior: (a) exposure HT; (b) exposure FT 


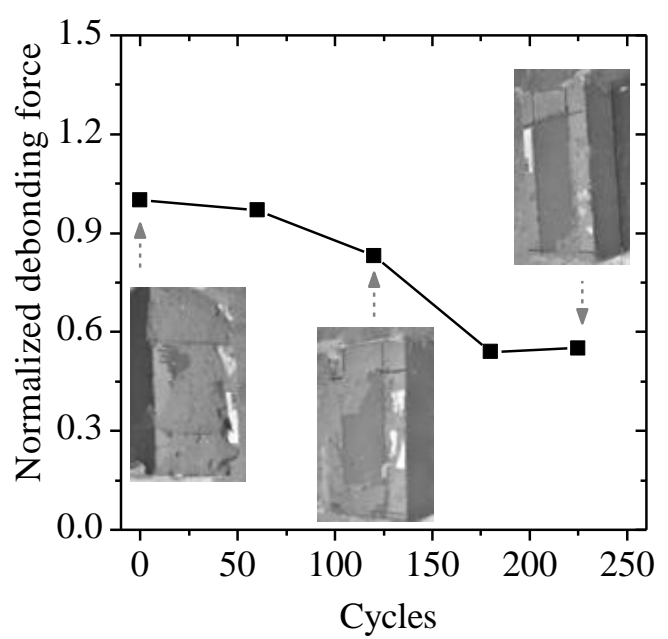

(a)

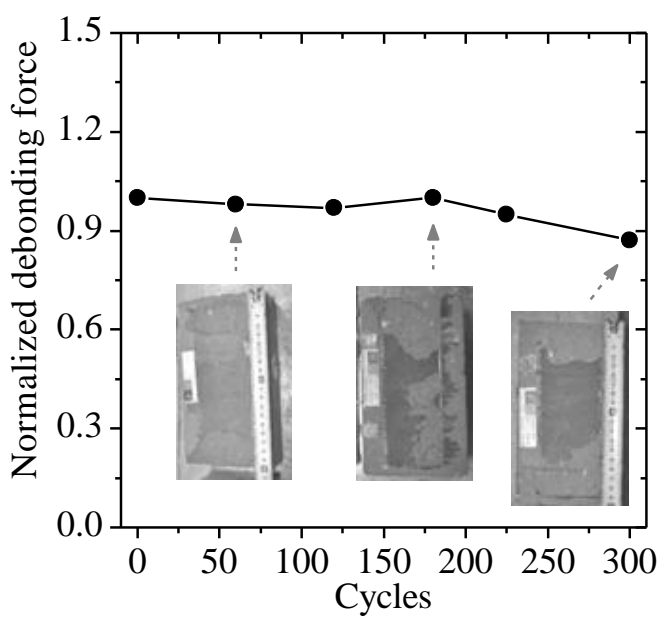

(b)

Fig. 13 Observed failure modes in shear bond tests: (a) exposure HT; (b) exposure FT 


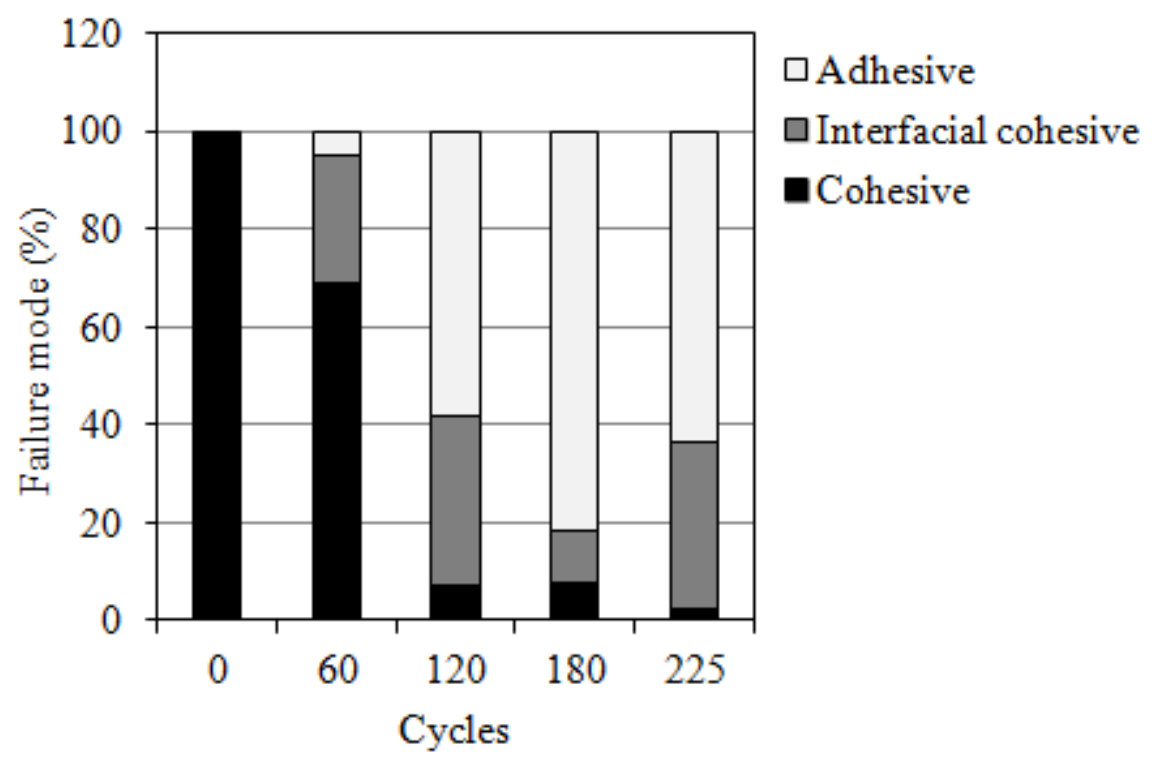

(a)

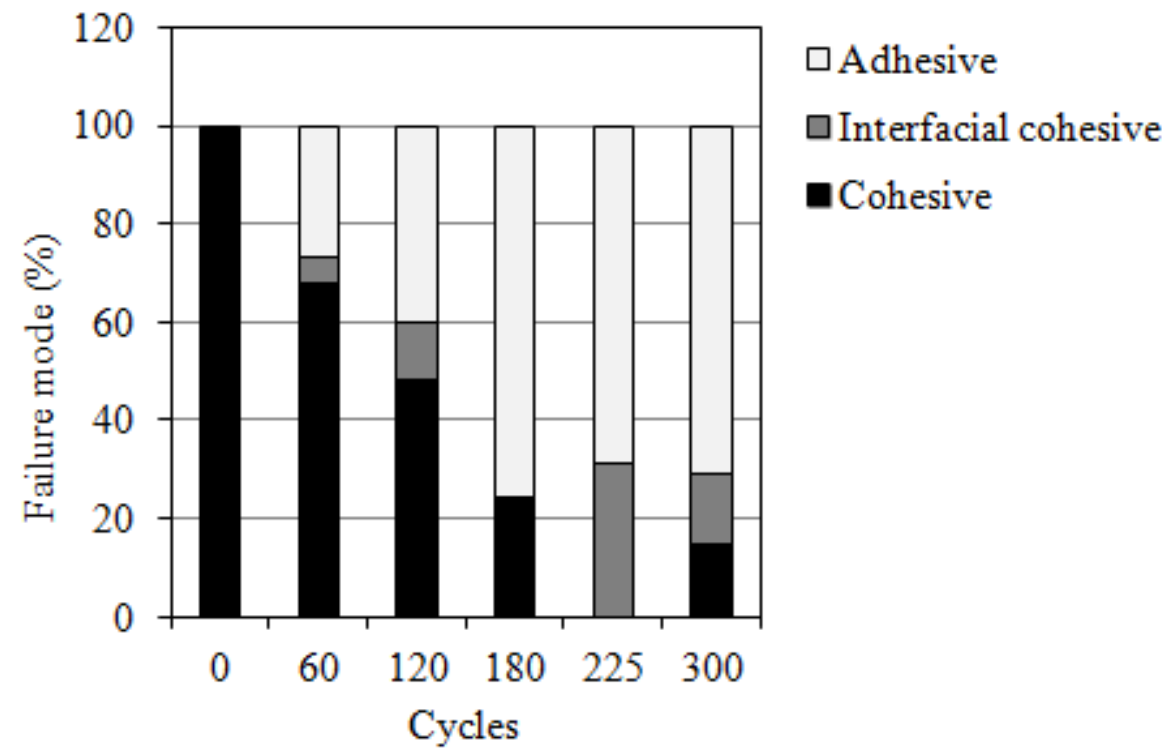

(b)

Fig. 14 Contribution of each failure mode in the debonding behavior: (a) exposure HT; (b) exposure FT 